\title{
Clonic hemifacial spasm from posterior fossa arteriovenous malformation
}

\author{
A DR I A PIERR Y A D MALCOLM CAMERON \\ From the Departments of Neurology and Neurosurgery, Salford Royal Hospital, Salford
}

SUMMARY A 21 year old woman presented with a two year history of right sided clonic hemifacial spasm. Investigations showed a large arteriovenous malformation on the right side of the posterior fossa and surgical exploration was undertaken. Early follow-up at six months showed a complete cure.

Over the last 30 years an increasing volume of evidence has been presented to support a structural vascular aetiology of hemifacial spasm (Campbell and Keedy, 1947; Gardner and Sava, 1962; Jannetta et al., 1977; Fabinyi and Adams, 1978; Maroon, 1978).

Maroon (1978), reviewing the literature, found 16 communications reporting a total of 107 cases of hemifacial spasm seemingly caused by vascular compression of the facial nerve. Confirmation in these cases was by surgery ( 92 cases), angiography (12 cases), and necropsy (three cases). In these cases the facial nerve compression appeared to be the result of normal, tortuous, ectatic, or redundant blood vessels (91 cases), aneurysm (11 cases), arteriovenous malformation (four cases), and primitive persistent aortic artery (one case).

We wish to report a further case of posterior fossa arteriovenous malformation apparently causing classical hemifacial spasm in a 21 year old woman, and complete relief of symptoms after microsurgical exploration of the posterior fossa.

\section{Case report}

A 21 year old, right handed female teacher presented in March 1978 with a two year history of right sided facial twitching. Initially only the external angle of the eye was involved but there was gradual spread over the next 12 months to involve the rest of the right side of the face although the forehead and platysma were spared. Stressful situations increased both the intensity

Address for correspondence and reprint requests: $\mathrm{Mr}$ Malcolm Cameron, Department of Neurosurgery, Pinderfields General Hospital, Aberford Road, Wakefield, West Yorkshire WF1 4DG.

Accepted 30 January 1979 and frequency of the twitchings while periods of relaxation might be associated with a marked improvement, and there was a complete remission for three weeks after success in her university examinations. During the six months before presentation she had experienced the tonus phenomenon with sustained facial contraction and eye closure lasting several seconds, associated with mild facial ache. There were no other symptoms.

The only abnormalities on examination were the features of right sided clonic hemifacial spasm including a mild facial weakness. It was interesting to observe that over short periods of time the facial twitching was synchronous with the peripheral pulse rate.

Investigations showed a normal full blood count, biochemical profile, electrocardiogram, and chest and plain skull radiographs. Computed tomography revealed an irregular area of high density on the right side of the posterior fossa extending into the cerebellopontine angle and up to the level of the tentorial hiatus. Vertebral angiography (Figs. 1 and 2) displayed a large and complex arteriovenous malformation on the right side of the posterior fossa. There were multiple arterial feeding vessels, the largest of which appeared to be an enlarged and tortuous right anterior inferior cerebellar artery.

Surgical exploration of the posterior fossa revealed a tight, clamp-like compression of the right facial nerve by two large abnormal arterial trunks. These offending arteries were divided between clips and then held away from the nerve with the aid of small soft prostheses. No attempt was made to excise the arteriovenous malformation. Postoperatively the patient made an uneventful recovery and was immediately completely free from 


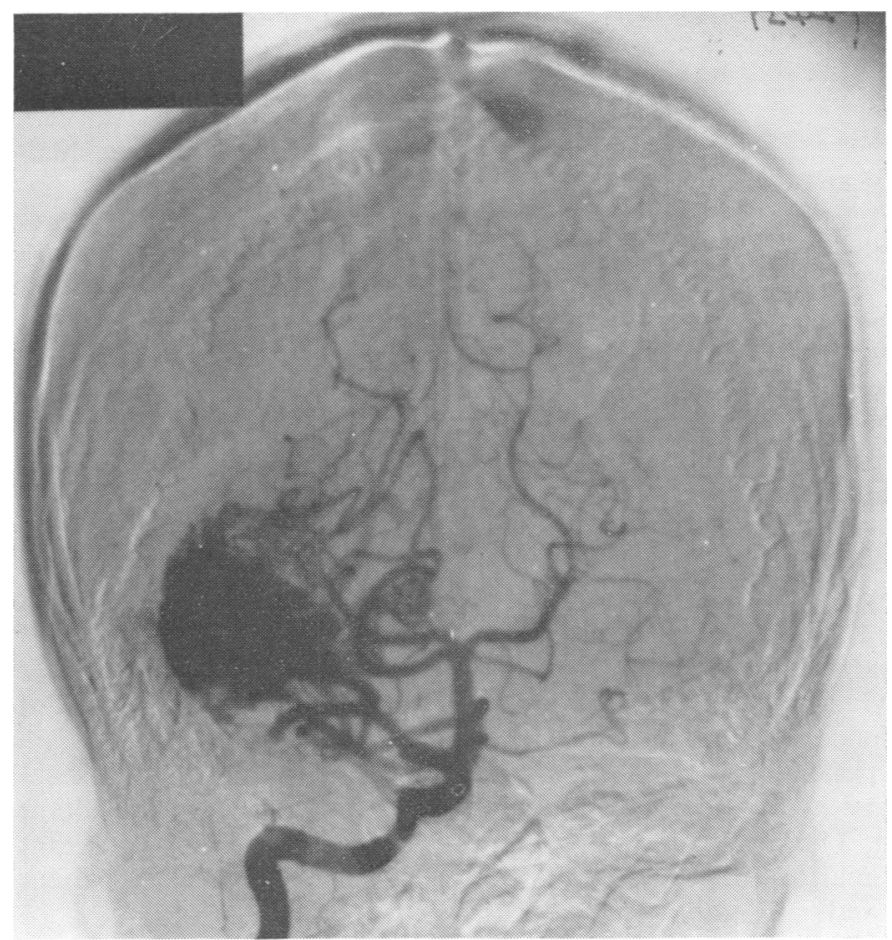

Fig. 1 Right vertebral, catheter arteriogram, A-P view, subtraction film, mid-arterial phase. The maljormation is supplied by at least five arteries from the vertebrobasilar system.

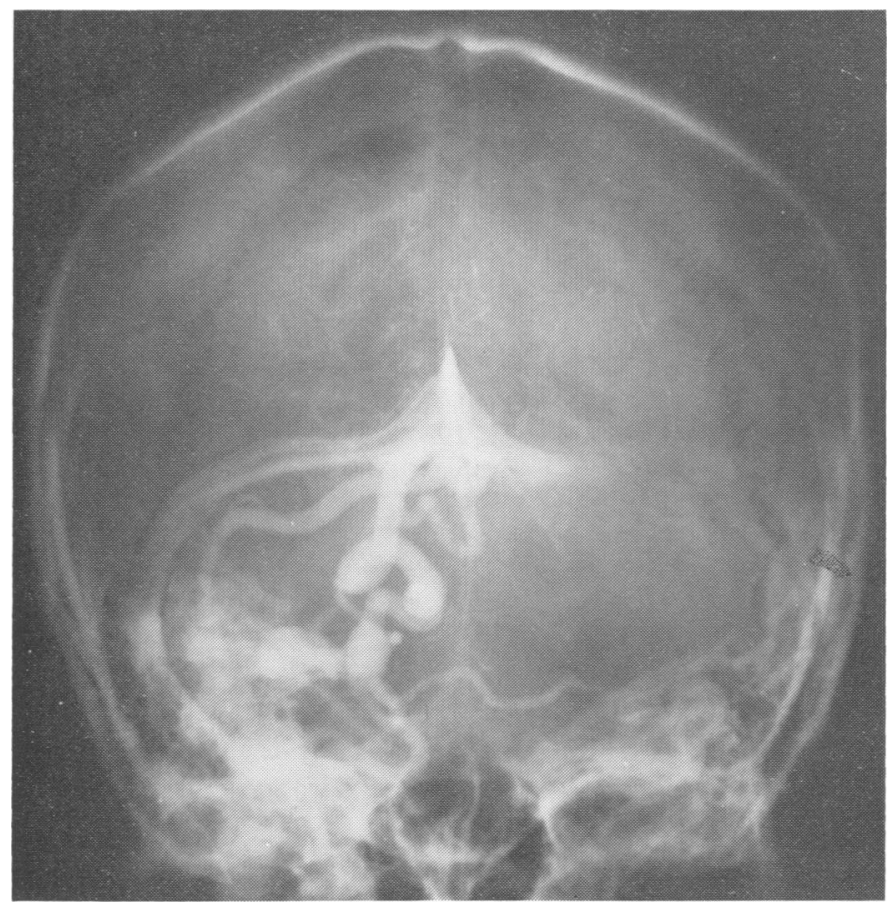

Fig. 2 Venous phase of the same arteriogram as shown in Fig. 1. 
spasm. Six months later she remained spasm-free with no abnormal neurological signs.

\section{Discussion}

In their review of 106 cases of hemifacial spasm from the records of the Mayo Clinic, Ehni and Woltman (1945) described the development of the disease as a distinct entity, and assessed the available evidence concerning its aetiology. They concluded: "it appears then, that the lesion is somewhere between the facial nucleus and the stylomastoid foramen. Thorough histological examination of this segment of the facial pathway in a victim of hemifacial spasm may settle the question."

Campbell and Keedy (1947) reported two patients both with hemifacial spasm and trigeminal neuralgia on the same side. At operation for the neuralgia each patient was found to have a basilar aneurysm compressing the facial nerve. Gardner and Sava (1962) provided operative evidence of facial nerve compression in 14 of their 19 patients with hemifacial spasm and obtained a very high cure rate after posterior fossa exploration. Three of their patients had an arteriovenous malformation in the cerebellopontine angle and all obtained complete relief. Jannetta et al. (1977) reported a large series of 47 patients treated by posterior fossa exploration and microsurgical decompression of the facial nerve, and presented convincing evidence that vascular compression of the facial nerve is truly aetiological in hemifacial spasm. However, Fabinyi and Adams (1978) have recently reported nine patients in six of whom exploration of the cerebellopontine angle revealed no abnormality. Nevertheless five of these six patients obtained complete relief of spasm and the remaining patient was greatly improved. They suggested that relief of spasm in these patients was due to the fibrosis generated by the trauma of placing Ivalon sponge around the facial nerve. This view was felt to be supported by the delay of several weeks in the complete abolition of the spasm which was seen in two patients. On the other hand, Jannetta et al. (1977) found that if they had stroked or manipulated the nerve at operation the patient usually awoke completely and permanently free of spasm.
The present patient is unusual in two respects -firstly the young age of onset (one of Jannetta's 45 patients with classical spasm was younger), and secondly, the association of hemifacial spasm with arteriovenous malformation. Arteriovenous malformation in the posterior cranial fossa is uncommon, and there appear to be only four previously reported cases of its association with hemifacial spasm (Gardner and Sava, 1962; Jannetta et al., 1977).

In our patient the CAT scan was very suggestive of arteriovenous malformation and, therefore, it was logical to proceed to vertebral angiography. In the patient with classical hemifacial spasm and normal CAT scan (including contrast enhancement) further neuroradiological investigations may be inappropriate and the question of therapy remains. Should the patient wish surgical treatment because of cosmetic, financial, or psychological disability it seems likely that the procedure of choice should be a microsurgical exploration of the cerebellopontine angle.

We wish to thank Dr P. D. Mohr, Consultant Neurologist, and Mr R. A. C. Jones, Consultant Neurosurgeon for permission to report this patient admitted under their care.

\section{References}

Campbell, E., and Keedy, C. (1947). Hemifacial spasm: a note on the aetiology in two cases. Journal of Neurosurgery, 4, 342-347.

Ehni, G., and Woltman, H. W. (1945). Hemifacial spasm. Review of one hundred and six cases. Archives of Neurology and Psychiatry (Chicago), 53, 205-211.

Fabinyi, G. C. A., and Adams, C. B. T. (1978). Hemifacial spasm: treatment by posterior fossa surgery. Journal of Neurology, Neurosurgery, and Psychiatry, 41, 829-833.

Gardner, W. J., and Sava, G. A. (1962). Hemifacial spasm-a reversible pathophysiologic state. Journal of Neurosurgery, 19, 240-247.

Jannetta, P. J., Abbasy, M., Maroon, J. C., Ramos, F. M., and Albir, M. S. (1977). Aetiology and definitive microsurgical treatment of hemifacial spasm. Journal of Neurosurgery, 47, 321-328.

Maroon, J. C. (1978). Hemifacial spasm. A vascular cause. Archives of Neurology (Chicago), 35, 481483. 